\section{Leitlinienkonformität der Versorgung von Brustkrebspatientinnen in Deutschland}

\author{
Im Jahre 2003 wurden in Deutschland erstmalig evidenz- und \\ konsensusbasierte Stufe-3 (S-3)-Leitlinien zum Thema Brust- \\ krebs publiziert und in 2008 aktualisiert [1, 2]. Sie weisen ne- \\ ben den klinischen Behandlungsempfehlungen Qualitätsindika- \\ toren aus. Diese überprüfen, ob die Empfehlungen in der prak- \\ tischen Versorgung der Patientinnen tatsächlich umgesetzt \\ werden. Entwickelt wurden sie durch die Leitlinienarbeitsgrup- \\ pen in Kooperation mit den wissenschaftlichen Mitarbeitern \\ der Bundesgeschäftsstelle Qualitätssicherung (BQS gGmbH). \\ A. Reiter ${ }^{1}$, D. Wallwiener ${ }^{2}$, M. Dombrowski ${ }^{3}$, W. Schleiz ${ }^{4}$, U. S. \\ Albert $^{5}$
}

\section{Einleitung}

Qualitätsindikatoren sind Kennzahlen, die - bezogen auf eine definierte Grundgesamtheit - qualitätsrelevante Parameter ausweisen. Als solche sind sie häufig mit einem Referenz- oder Zielwert versehen. So kann die Qualität der bewerteten Versorgung mit dem zu erreichenden Zielwert verglichen werden.

Die BQS hat die Leitlinien-Indikatoren für die praktische Anwendung im stationären
Sektor einschließlich ihrer methodischen Überprüfung weiter entwickelt und bis einschließlich 2009 in Form von bundesweit einheitlichen Dokumentationsvorgaben umgesetzt. Ziel war ein zunächst anonymer Qualitätsvergleich zwischen allen an der Versorgung von Brustkrebspatientinnen beteiligten Krankenhäusern. Bei diesen Aufgaben wurde die BQS von einem interdisziplinären Team an Fachexperten einschließlich Patientenvertreterinnen unterstützt. Die Beteiligung von Leitlinienautoren gewährleistete eine Ko- härenz und einen kontinuierlichen Austausch zwischen Leitlinien und der vergleichenden Qualitätssicherung. Seit 2006 müssen alle Krankenhäuser die Ergebnisse ausgewählter Indikatoren publizieren.

\section{BQS-Indikatoren}

Die BQS hat im Bereich Brustkrebs insgesamt 6 Indikatoren zur Prozessqualität und 4 weitere zur Qualität der Indikationsstellung eingesetzt ( $\odot$ Tab. 1 ). Alle Indikatoren beziehen sich auf Statements aus den genannten Leitlinien. In 2008 mussten alle Krankenhäuser die Öffentlichkeit über die Ergebnisse der 3 Indikatoren „Intraoperatives Präparatröntgen“, „Hormonrezeptor- und HER-2/neu-Analyse“ und „Angabe Sicherheitsabstand“ informieren. Vor der Festlegung dieser Indikatoren für die öffentliche Berichterstattung wurde deren methodische Qualität umfassend durch die BQS geprüft. Zu diesem Zweck wurde eigens ein Instrument namens QUALIFY entwickelt [3]. Nur methodisch hochwertige Indikatoren sind geeignet, die Öffentlichkeit adäquat zu informieren. Indikatoren, die den QUALIFY-Test nicht bestanden haben, liefern zwar auch wichtige Informationen zur Qualität der Versorgung, bedürfen aber zusätzlicher Kenntnisse - zum Beispiel des Case-mix eines Krankenhauses -, um die Ergebnisse angemessen zu interpretieren.

Die Qualität der Versorgung von Brustpatientinnen im Behandlungszeitraum 2008 wird exemplarisch anhand der 3 berichtspflichtigen Indikatoren dargestellt. Aus Gründen der statistischen Aussagekraft

\begin{tabular}{|c|c|c|}
\hline BQS-Indikatoren 2008 & Gesamtergebnisse & Referenzwerte \\
\hline \multicolumn{3}{|l|}{ Indikationsqualität } \\
\hline Prätherapeutische histologische Diagnosesicherung & $89,8 \%$ & $\geq 70 \%$ \\
\hline Primäre Axilladissektion bei DCIS & $4,3 \%$ & $\leq 5 \%$ \\
\hline Indikation zur Sentinel-Lymphknoten-Biopsie & $81,3 \%$ & $\geq 63,5 \%$ \\
\hline Indikation zur brusterhaltenden Therapie & $82,8 \%$ & $\geq 60 \%$ und $\leq 85 \%$ \\
\hline \multicolumn{3}{|l|}{ Prozessqualität } \\
\hline Intraoperatives Präparatröntgen & $94,2 \%$ & $\geq 95 \%$ \\
\hline \multicolumn{3}{|l|}{ Hormonrezeptoranalyse und HER-2/neu-Analyse... } \\
\hline Hormonrezeptoranalyse & $98,9 \%$ & $\geq 95 \%$ \\
\hline Her-2/neu-Analyse & $98,0 \%$ & $\geq 95 \%$ \\
\hline \multicolumn{3}{|l|}{ Angabe Sicherheitsabstand... } \\
\hline bei brusterhaltender Therapie & $96,1 \%$ & $\geq 95 \%$ \\
\hline bei Mastektomie & $93,2 \%$ & $\geq 95 \%$ \\
\hline Anzahl Lymphknoten & $92,3 \%$ & $\geq 95 \%$ \\
\hline Meldung an Krebsregister & $90,9 \%$ & $\geq 95 \%$ \\
\hline Zeitlicher Abstand zwischen Diagnose und Operationsdatum & 13,0 Tage & $\geq 6,0$ und $\leq 20,0$ Tage \\
\hline
\end{tabular}




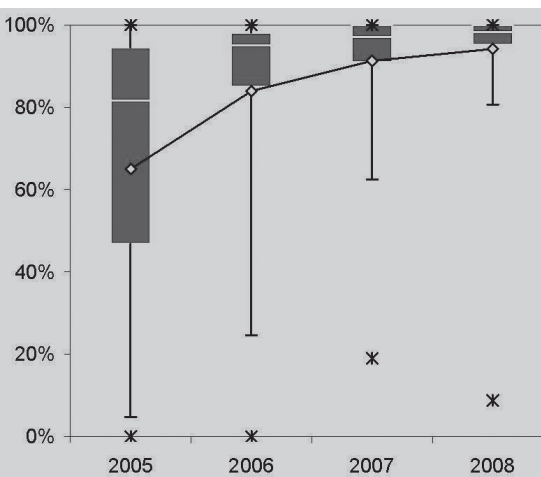

Abb. 1 Verlaufsdarstellung Intraoperatives Präparatröntgen durch Box-and-Whisker-Plot (Gesamtergebnisse aller Patientinnen: Rauten; Verteilung der Krankenhausergebnisse mit mindestens 20 Fällen: Balken begrenzt durch $25 \%$ - und $75 \%$-Perzentil, weiße Linien: Median, Whiskers begrenzt durch $5 \%$ - und $95 \%$ Perzentil, Sterne: Minimum und Maximum).

werden in diesem Artikel Ergebnisse für Krankenhäuser nur dann dargestellt, wenn mindestens 20 Fälle in der Grundgesamtheit des jeweiligen Indikators erreicht werden. Selbstverständlich gelten auch für Krankenhäuser mit kleinen Fallzahlen gleiche Qualitätsanforderungen in der praktischen Umsetzung der Qualitätssicherung.

\section{Intraoperatives Präparatröntgen \\ $\nabla$}

Dieser Indikator misst, ob bei stationären Patientinnen mit nicht tastbaren Brusttumoren und präoperativer Befundmarkierung unter mammografischer Sicht, intraoperativ die vollständige Tumorentfernung durch ein Präparatröntgen geprüft wird. In 94,2 \% der Fälle in Deutschland wurde ein Präparatröntgen dokumentiert. Die Gesamtergebnisse zu diesem Indikator haben sich in den vergangenen Jahren in beeindruckender Weise verbessert, bei gleichzeitig abnehmender Varianz (Gesamtergebnisse 2005: 65,0\%, 2006: 83,9\%, 2007: 91,2\%) (॰Abb. 1).

Ein Blick auf die Prozessleistung der einzelnen Krankenhäuser zeigt, dass 61 von 283 Kliniken mit mindestens 20 radiologisch gesteuerten Draht-Markierungen den Referenzbereich von mindestens 95\% nicht erreichten (๑Abb. 2).

\section{Angabe Sicherheitsabstand}

Dieser Indikator misst, ob der Pathologe den minimalen Abstand zwischen dem Karzinom und dem Resektionsrand exakt in mm angibt. In 96,1\% aller Fälle mit brusterhaltender Therapie (BET) und in 93,2\% der Fälle mit Mastektomie wurde 2008 ein metrischer Sicherheitsabstand dokumentiert. Die Versorgung hat sich im Vergleich zum Vorjahr (92,9 bzw. 88,1\%) signifikant verbessert. Erstmalig wurde der Referenzbereich von mindestens 95\% für den Sicherheitsabstand bei BET auf Bundesebene erreicht. Die auffälligsten Krankenhausergebnisse lagen bei 22,7 bzw. 23,1\%. 120 von 499 bzw. 133 von 388 Krankenhäusern (mit mindestens 20 Fällen in der jeweiligen Grundgesamtheit BET bzw. Mastektomie) erreichten den Referenzbereich nicht.

\section{Hormonrezeptoranalyse und HER-2/neu-Analyse \\ $\nabla$}

Dieser Indikator hat 2008 ergeben, dass bei $98,9 \%$ aller Patientinnen mit invasivem Mammakarzinom die Hormonrezeptoren immunhistochemisch analysiert wurden. 553 von 577 Krankenhäusern mit einer Behandlung von mindestens 20 Primärerkrankungen oder Rezidiven erreichten den Referenzbereich von 95\%. Die erzielten Ergebnisse aller Krankenhäuser lagen dabei zwischen $25,0 \%$ bis $100,0 \%$.

Her-2/neu wurde bei 98,0\% aller Patientinnen mit einer Primärerkrankung bestimmt. Im bundesweiten Durchschnitt wurde der Referenzbereich von mindestens 95\% also erreicht. Auf der Ebene der Krankenhäuser haben 520 von 569 mit mindestens 20 Primärerkrankungen die Vorgaben erfüllt. Das schlechteste Krankenhaus-Ergebnis betrug 23,8\%.

Wie kommen diese positiven Verläufe zustande? In den vergangenen Jahren sind

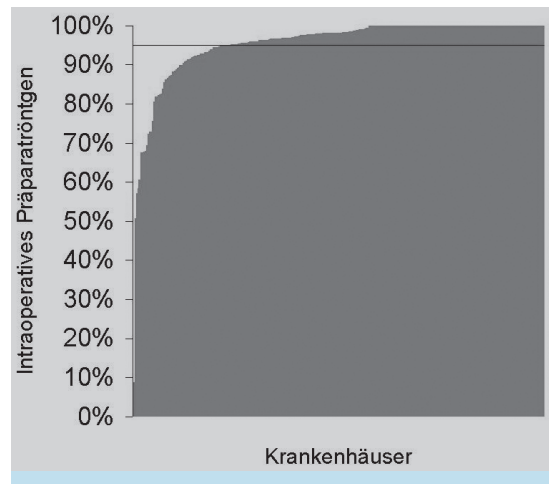

Abb. 2 Rate an intraoperativen Präparatröntgen für 283 Krankenhäuser mit $\geq 20$ Drahtmarkierungen durch Mammografie. eine ganze Reihe von Maßnahmen zur Verbesserung der Versorgungsqualität auf den Weg gebracht worden. Von großer Bedeutung ist die Verfügbarkeit der S-3-Leitlinien, auf die sich weitere Interventionen wie der Strukturierte Dialog mit auffälligen Krankenhäusern, Zertifizierungsverfahren und Anstrengungen im Rahmen des internen Qualitätsmanagements explizit beziehen können. Im Falle flächendeckend nicht zufriedenstellender Indikatorergebnisse sind die BQS und ihre Fachexperten mit den zuständigen wissenschaftlichen Fachgesellschaften in einen Dialog getreten. Strategien zur effizienten Umsetzung der betroffenen Leitlinienempfehlungen wurden vereinbart. Nicht zuletzt hat die BQS durch die Publikation und Diskussion der Ergebnisse im jährlichen Qualitätsreport und auch auf zahlreichen Fachkongressen eine kontinuierliche Qualitätsverbesserung mit unterstützt.

\section{Diskussion \\ $\nabla$}

Anhand der ausgewerteten Indikatoren konnte im Verlauf der vergangenen Jahre eine kontinuierliche Verbesserung der Prozessparameter für die Versorgung von Brustkrebspatientinnen, gemessen an den Vorgaben der S-3-Leitlinien, festgestellt werden. Trotz dieser positiven Nachricht gibt es weiterhin eine gewisse Anzahl an Krankenhäusern, die die Zielwerte nicht erreichen. Hier sind weitere Anstrengungen aller an der Qualitätsverbesserung Beteiligten unbedingt erforderlich.

Bei der Darstellung der erreichten Versorgungsqualität durch die BQS vermissen insbesondere Patientinnen die Darstellung der für ihre Krankenhauswahl relevanten Ergebnisparameter Mortalität, Rezidive und Lebensqualität. Diese sind in der Tat wichtige Qualitätsdaten. Auch fehlen Angaben aus dem ambulanten Bereich wie z. B. der Qualität der adjuvanten Therapie. Die Zukunft liegt in einer sektorenübergreifenden Qualitätssicherung der gesamten Versorgungskette - von der Früherkennung bis zur Nachsorge - unter Einbeziehung von Ergebnisindikatoren.

Aber auch im Falle der Verfügbarkeit von Mortalitätsdaten bleiben Prozessindikatoren wichtigste Bausteine der Qualitätsförderung. Bei diesen muss die Evidenzbasierung gewährleistet sein, d. h. die abgebildeten Prozesse müssen relevante Versorgungsergebnisse wie Mortalität, Rezidive und Lebensqualität maßgeblich beeinflus- 
sen. Der Nachweis dieses Zusammenhanges wird in den S-3-Leitlinien, die Empfehlungen für bestimmte Behandlungen (d. h. Prozesse) geben, explizit auf der Grundlage wissenschaftlicher Studienergebnisse und von klinischem Sachverstand erbracht. Ein wesentlicher Vorteil von Prozessindikatoren besteht unzweifelhaft darin, dass erforderliche Qualitätsverbesserungen einfacher und schneller identifiziert werden können. Bei Ergebnisindikatoren wie der Mortalität ist eine Ursachenklärung unbefriedigender Krankenhausergebnisse häufig nicht mehr möglich: Die Behandlung liegt meistens viele Jahre zurück, eine meist sehr aufwändige Risikoadjustierung muss Einflussfaktoren wie das ursprüngliche Krankheitsstadium, Alter und Begleiterkrankungen berücksichtigen. Nicht zuletzt sind die notwendigen Konsequenzen aus Mortalitätsunterschieden häufig nicht eindeutig, da sich das therapeutische Vorgehen durch eine Aktualisierung der Empfehlungen in der Zwischenzeit ohnehin verändert hat. Daraus folgt, dass Prozess- und Ergebnisindikatoren 2 sich ergänzende, nicht aber konkurrierende, Sichtweisen auf die Qualität der Versorgung sind.

\section{Fazit}

Die Qualitätsindikatoren aus den S-3-Leitlinien zur Erkennung bzw. Behandlung von Brustkrebs konnten in den gemessenen Bereichen eine kontinuierliche Verbesserung der Versorgung von Brustkrebspatientinnen belegen. Nächstes Ziel muss eine Ergänzung der Qualitätsdarstellung über die Sektorengrenzen hinaus unter Einbeziehung von Ergebnisparametern sein. Die Praxis hat gezeigt, dass Leitlinien und Qualitätsindikatoren exzellente Partner auf dem Wege der Qualitätsförderung sind.

\section{Literatur}

1 Albert U-S. Stufe-3-Leitlinie BrustkrebsFrüherkennung in Deutschland, 1. Aktualisierung 2008. München: Zuckschwerdt Verlag, 2008

2 Kreienberg R, Kopp I, Albert U et al. Interdisziplinäre S3-Leitlinie für die Diagnostik, Therapie und Nachsorge des Mammakarzinoms. In: Deutsche Krebsgesellschaft e.V., Informationszentrum für Standards in der Onkologie (ISTO) (Hrsg). Berlin, 2008

3 Reiter A, Fischer B, Kötting J et al. QUALIFY: Ein Instrument zur Bewertung von Qualitätsindikatoren. Z Evid Fortbild Qual Gesundhwes 2008; 101(10): 683-88
Institutsangaben

1 Abteilung Medizin und Pflege, BQS Bundesgeschäftsstelle Qualitätssicherung gGmbH, Düsseldorf

\section{Universitäts-Frauenklinik, Tübingen}

3 Klinik für Frauenheilkunde und $\mathrm{Ge}$ burtshilfe, Ev. Waldkrankenhaus Spandau, Berlin

4 Abteilung Medizinische Biometrie, BQS Bundesgeschäftsstelle Qualitätssicherung gGmbH, Düsseldorf

5 Klinik für Frauenheilkunde und Geburtshilfe, Frauenklinik der Philipps-Universität, Marburg

\section{Korrespondierende Autorin}

Dr. med. Anne Reiter

$B Q S$ Bundesgeschäftsstelle Qualitäts-

sicherung $\mathrm{gGmbH}$

Kanzlerstr. 4

40472 Düsseldorf

E-Mail: a.reiter@bqs-institut.de 